\title{
Periductal Breast Myoepitheliosis
}

National Cancer Institute

\section{Source}

National Cancer Institute. Periductal Breast Myoepitheliosis. NCI Thesaurus. Code C40388.

A neoplastic process characterized by the proliferation of spindle to cuboidal myoepithelial cells around small breast ducts. 